\title{
Incidencia del trabajo colaborativo de docentes universitarios en el contexto de la acreditación internacional
}

\author{
Incidence of Collaborative Work of University Teachers in the Context of \\ International Accreditation
}

\section{Incidência de trabalho colaborativo de professores universitários no contexto de acreditação internacional}

\section{Guadalupe González García}

Universidad Autónoma del Estado de México, México

ggonzalezga@uaemex.mx https://orcid.org/0000-0002-4119-1187

\section{Resumen}

En las organizaciones educativas el factor más importante es el humano, clave para el cambio y el mejoramiento. El presente documento busca respuestas a la siguiente pregunta: ¿cómo podría el trabajo colaborativo entre los docentes universitarios incidir de manera positiva en los procesos de acreditación de los programas educativos? Esta investigación bibliográfica de carácter expositivo primeramente aborda el trabajo colaborativo entre universitarios; continúa con la evolución de la acreditación en México y en el contexto internacional, y finalmente propone algunas acciones para vincular a ambos, trabajo colaborativo y acreditación, con la calidad educativa. Los procesos de acreditación de programas educativos demandan que los trabajos colaborativos entre docentes cumplan con la necesidad de compartir el conocimiento e incrementarlo. Los resultados llevan a las premisas recomendadas: 1) difundir los productos y logros entre los integrantes de la propia comunidad universitaria; 2) publicar los resultados a través de las redes de investigación de las universidades; 3) abordar la innovación articulando procesos creativos; 4) trascender en la 

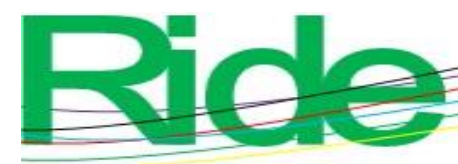

Revista Iberoamericana para la Investigación y el Desarrollo Educativo

ISSN 2007 - 7467

coherencia de contenidos y 5) contribuir a la calidad de los programas. Por último, se considera que sería importante contar con esquemas de seguimiento para medir y evaluar el impacto de los trabajos colaborativos en la calidad de la educación, acorde a los parámetros de acreditación internacional.

Palabras clave: acreditación; calidad de la educación, docencia, trabajo colaborativo.

\section{Abstract}

In educational organizations, the most important factor is the human, key to change and improvement. This document seeks an answer to the following question: how could collaborative work between teachers have a positive influence on accreditation processes of educational programs? This expository bibliographic research first addresses the collaborative work between university students; continues with the evolution of accreditation in Mexico and in the international context, and finally proposes some actions to link them with educational quality. The accreditation processes of educational programs requires that collaborative work among teachers respond to the need to share knowledge and increase it. The recommended premises are 1) dissemination of the products and achievements among the members of the university community itself; 2) publish the results through the research networks of the universities; 3) address innovation by articulating creative processes; 4) transcend the coherence of content, and 5) contribute to the quality of the programs. Finally, it would be important to have monitoring schemes to measure and evaluate the impact of collaborative work on the quality of education, according to the international accreditation parameters.

Keywords: accreditation, quality of education, teaching, collaborative work. 


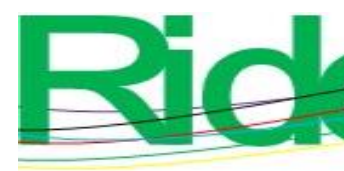

Revista Iberoamericana para la Investigación y el Desarrollo Educativo ISSN $2007-7467$

\section{Resumo}

Nas organizações educacionais, o fator mais importante é o humano, chave para a mudança e o aprimoramento. Este documento busca respostas para a seguinte questão: como o trabalho colaborativo entre professores universitários pode influenciar positivamente os processos de acreditação de programas educacionais? Esta pesquisa bibliográfica expositiva aborda primeiro o trabalho colaborativo entre estudantes universitários; continua com a evolução da acreditação no México e no contexto internacional e, finalmente, propõe algumas ações para vincular o trabalho colaborativo e a acreditação com a qualidade educacional. Os processos de acreditação de programas educacionais exigem que o trabalho colaborativo entre os professores atenda à necessidade de compartilhar conhecimentos e aumentá-los. Os resultados levam às premissas recomendadas: 1) divulgar os produtos e realizações entre os próprios membros da comunidade universitária; 2) divulgar os resultados por meio das redes de pesquisa das universidades; 3) abordar a inovação articulando processos criativos; 4) transcender a coerência do conteúdo e 5) contribuir para a qualidade dos programas. Por fim, considera-se que seria importante contar com esquemas de monitoramento para medir e avaliar o impacto do trabalho colaborativo na qualidade da educação, segundo parâmetros internacionais de acreditação.

Palavras-chave: acreditação; qualidade da educação, ensino, trabalho colaborativo.

Fecha Recepción: Marzo 2020

Fecha Aceptación: Enero 2021

\section{Introducción}

Las acreditaciones de programas académicos a nivel universitario avalan el reconocimiento de la calidad de los programas educativos, como acotan Bodero (2014) y Elías (2015). Como parte del proceso de acreditación, además, se transparenta información sustantiva de toda institución de educación superior (IES), a saber: servicios, procesos, procedimientos académicos y administrativos, fuentes de financiamiento, entre otros (Consejo para la Acreditación de la Educación Superior [Copaes], 2019a). Según la Asociación Nacional de Universidades e Instituciones de Educación Superior [Anuies] (2019), la acreditación se origina con el fin de mantener los niveles de calidad en la enseñanza. 

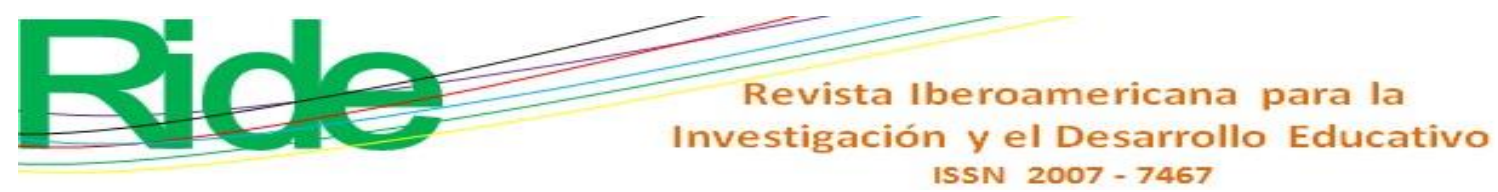

La acreditación, discurren Jiménez (2013) y Mendoza y Ortegón (2019), ha evolucionado a tal punto que ahora, más allá de considerarse una opción que puede tomar una IES, es ya un compromiso casi obligatorio para adoptar estándares de calidad cada vez más altos y entregar resultados concretos que se puedan validar y valorar. Su principal reto es ofrecer una educación consistente, donde los fundamentos de un programa educativo y sus resultados sean coherentes entre sí y además ofrezcan respuestas tangibles ante la demanda laboral en la que se insertarán sus egresados. Para ello, las funciones y responsabilidades del personal docente y administrativo deben ser claras y dirigidas al cumplimiento de los objetivos de cada programa educativo.

En el contexto actual, los programas educativos tienen la necesidad de acreditarse no solo localmente, sino a nivel internacional (Rama, 2009). Esta necesidad marca el derrotero de las diversas actividades académicas y administrativas de las instituciones e incide en la toma de decisiones fundamentales que buscan asegurar una mayor calidad en la educación (Organización de las Naciones Unidas para la Educación, la Ciencia y la Cultura [Unesco], 2015). Asimismo, una acreditación internacional aumenta la competencia, puede mejorar la cooperación y el intercambio internacional, facilita la educación transfronteriza, mejora la legitimidad de las instituciones educativas y facilita la comparación internacional (Miranda, 2019).

En cualquier organización el recurso más importante es precisamente el humano, que es clave para el cambio y el mejoramiento. A sabiendas de ello, el presente artículo busca respuesta a la siguiente pregunta: ¿cómo podría el trabajo colaborativo entre los docentes universitarios incidir de manera positiva en los procesos de acreditación de los programas educativos? Primeramente se aborda la conceptualización del trabajo colaborativo, para continuar con la evolución de la acreditación en México y en el contexto internacional. Finalmente, se proponen acciones para vincular el trabajo colaborativo y la acreditación internacional con la calidad educativa.

\section{El trabajo colaborativo de docentes universitarios}

El trabajo colaborativo, como lo define Maldonado (2007), es aquel que constituye un modelo de aprendizaje interactivo que invita a construir juntos, para lo cual demanda conjugar esfuerzos, talentos y competencias mediante una serie de transacciones que permitan lograr las metas establecidas en consenso. La misma autora agrega que "es el 


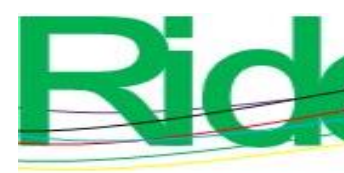

Revista Iberoamericana para la Investigación y el Desarrollo Educativo ISSN $2007-7467$

conjunto de métodos de instrucción o entrenamiento para uso en grupos, así como de estrategias para propiciar el desarrollo de habilidades mixtas: aprendizaje y desarrollo personal y social" (Maldonado, 2007, p. 165). Asimismo, refiere que sus características son:

1) Tiene la condicionante de que debe existir una reciprocidad entre los participantes.

2) Implica compartir experiencias y conocimientos.

3) Promueve la adquisición de destrezas y actitudes que ocurren como resultado de la interacción en grupo.

4) La construcción del aprendizaje consensuado es mediante la cooperación de los miembros del grupo

5) Se comparte la autoridad y entre todos se acepta la responsabilidad de las acciones del grupo.

Ahora bien, es necesario precisar que los trabajos colaborativos entre docentes surgen de la necesidad de compartir el conocimiento, incrementarlo e incluso favorecer la metacognición; han resultado en lo que se conoce como producción entre pares académicos. Los principios que los guían son compartidos por quienes toman parte de los proyectos y se considera que los integrantes son expertos o buenos conocedores de los temas que planean desarrollar en conjunto (López, 2015). La sociedad del conocimiento demanda enfrentar nuevos retos y oportunidades para que evolucione la educación superior: la colaboración académica es una herramienta para complementar habilidades y compartir experiencias entre universidades (Rodríguez, 2015).

El trabajo colaborativo entre docentes de diferentes países fue ampliamente considerado en la revisión de literatura de Ángel y Cano (2011). Se evidenció que uno de los procesos más complejos al abordar este tipo de proyectos es el mediacional que genere interacciones entre los participantes, teniendo en cuenta que se conforman entidades individuales o grupales de acuerdo con los propósitos. Al carecer del requisito de integrarse por cuerpo de investigación de manera formal, genera vínculos de mayor lealtad y promueve lazos de compromiso, correspondencia, fidelidad y camaradería.

En la era del conocimiento, tanto el Internet como las tecnologías de la información y comunicación (TIC) han facilitado nuevas formas de interactuar y de comunicarnos. Al evolucionar, surgió también una nueva forma o modelo de comportamiento académico donde a través de la colaboración entre miembros de diferentes comunidades educativas los productos finales se ven enriquecidos. 


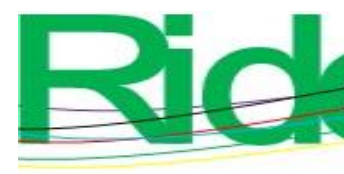

Revista Iberoamericana para la Investigación y el Desarrollo Educativo ISSN 2007 - 7467

Hoy en día, los trabajos colaborativos son cada vez más fáciles de promover, desarrollar, localizar, difundir y de ser vistos simultáneamente por muchas personas, sin importar el lugar donde se encuentren y prescindiendo de la cercanía física. Basta con localizar revistas científicas para encontrar artículos con autores de diferentes regiones trabajando juntos. Además, existen documentos cuyos contenidos están abiertos a modificarse, corregirse y enriquecerse, incluso por personas que no participaron en el proceso de su creación.

Ciertamente, como reflexiona Dorta (2016), una universidad es más conocida en el panorama internacional en la medida en que se hagan más visibles y medibles sus resultados en el terreno de la ciencia. Y esto, decididamente, genera un valor agregado a las producciones con calidad.

El trabajo colaborativo en la academia se distingue cuando existe una reciprocidad entre un conjunto de individuos que saben diferenciar y contrastar sus puntos de vista, de tal manera que llegan a generar un proceso de construcción de conocimiento (Revelo, Collazos y Jiménez, 2018). Ahora bien, López, García y Larios (2018) abundan en los factores que invitan a los investigadores a trabajar en esquemas colaborativos, entre los cuales se pueden mencionar la búsqueda por acceder a determinados grupos, la complementación de habilidades técnicas y dominios disciplinares, el reconocimiento de la mayor visibilidad e impacto, el apoyo a la movilidad y la formación de recursos humanos.

La experiencia nos dice que los grupos colaborativos no están plenamente regulados. Así que no necesariamente responden a una cuestión de investigación en curso que resulte atractiva para los participantes, ya sea que se trate de básica o aplicada. La colaboración tiende a ser espontánea, de interés mutuo y, muchas de las veces, más o menos explícita. De igual manera, ya sea por académicos de la misma o distinta organización educativa (intrainstitucionales o interinstitucionales), los temas se trabajan con más o menos profundidad. Y lo mismo sucede cuando se trata de enfoques locales o internacionales, a partir de un objetivo común, donde la respuesta debiera satisfacer a todos.

En esa línea, Domínguez, Crhová y Molina (2015) resumen la importancia de la aparición en los años 2000 de los cuerpos académicos —en el marco del Programa de Mejoramiento del Profesorado (Promep) de la Secretaría de Educación Pública (SEP), hoy Programa para el Desarrollo Profesional Docente, para el Tipo Superior (Prodep) — para organizar el trabajo académico, y reflexionan también sobre las diversas dinámicas de actuación de estos en las diferentes universidades de México. De manera muy breve, se alude 


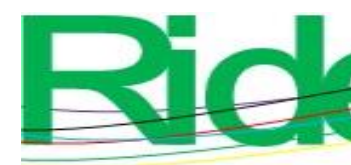

Revista Iberoamericana para la Investigación y el Desarrollo Educativo ISSN $2007-7467$

a que el objetivo de un cuerpo académico es que los profesores de tiempo completo, a través de grupos, trabajen colaborativamente de tal forma que impacten al interior de sus propias instituciones y comunidades con la generación de nuevo conocimiento.

Si bien se parte del supuesto de que los investigadores aporten esfuerzos hacia la consecución de ese objetivo en común, esto no siempre se cumple en la realidad, derivado de otros intereses más bien de tipo personal y de reconocimiento. Por citar un ejemplo, Bozeman y Craig (2013) hallaron que uno de los motivadores para la investigación colaborativa en cuerpos académicos tiene que ver más con la formación de capital humano, principalmente posgraduados, que con la aplicación innovadora del conocimiento. Paralelamente, Branco y Craig (2010) manifiestan, de acuerdo con los resultados de su investigación, la importancia de afiliar cuerpos académicos con centros de investigación universitaria mejoraría la productividad y facilitaría la colaboración de los docentes.

Al mismo tiempo, se vislumbra que una alternativa interesante para el trabajo colaborativo entre docentes universitarios sería la integración cada vez mayor de los cuerpos académicos a centros de investigación, ya que promovería la influencia en los modelos de colaboración y productividad de los investigadores, y facilitaría la participación interdisciplinaria e interinstitucional. Para finalizar este apartado, habría que concluir con que el trabajo colaborativo entre pares, al igual que el proveniente de los cuerpos académicos, se determina porque cada quien tiene sus alcances, su estructura, su registro puntual de hallazgos y compromisos.

\section{La acreditación de los programas educativos en México y el mundo}

La acreditación es un proceso mediante el cual una IES somete sus programas académicos a una evaluación con el fin de obtener por parte de un organismo no gubernamental, reconocido y avalado, el reconocimiento de que su oferta académica cumple con estándares de calidad. Por lo anterior, constituye una fuente confiable de credibilidad y confianza, como expresa el Consejo de Acreditación de Ciencias Administrativas, Contables y Afines [Caceca] (2018). Tiene su antecedente inmediato en los procesos de evaluación que adquirieron importancia creciente en todo el mundo a partir de la década de los años 80, según refiere Jiménez (2013). 


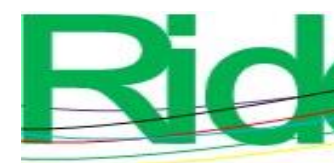

Revista Iberoamericana para la
Investigación y el Desarrollo Educativo ISSN 2007-7467

Organizaciones como la Unesco y la Organización para la Cooperación y Desarrollo Económicos (OCDE) han promovido a nivel internacional acciones encaminadas a la evaluación externa mediante un proceso de revisión que procura garantizar la calidad en las IES. Los programas académicos son evaluados con base en estándares y criterios previamente establecidos, y el proceso es llevado a cabo por un organismo externo reconocido por el Copaes (2019b) por medio del proceso de acreditación descrito en la figura 1.

Figura 1. Proceso de acreditación

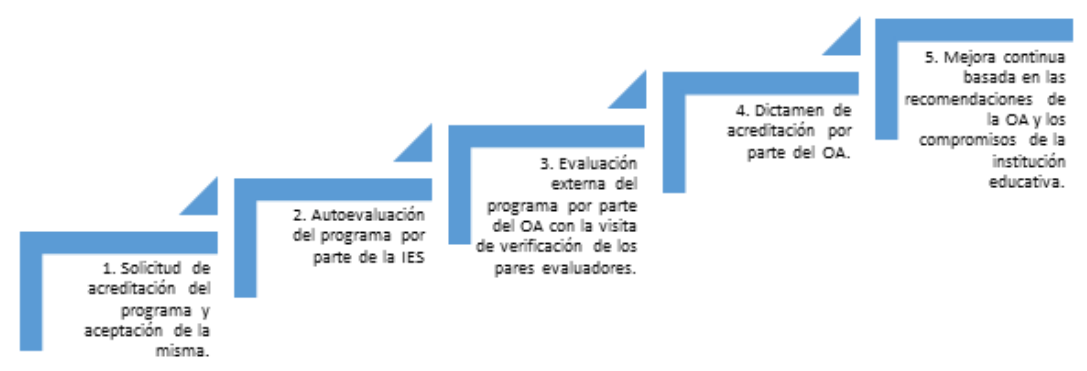

Fuente: Elaboración propia con base en Copaes (2019b)

Las acreditaciones universitarias buscan orientar a las instituciones educativas y transparentar la información acerca de sus servicios, fines, metodología, procedimientos, procesos, criterios, parámetros, productos, resultados, administración y fuentes de financiamiento. Los estándares que se determinan para acotar los puntos que requieren de mayor análisis y valoración en los programas de estudio son: gestión, formación, infraestructura, resultados y planeación.

Las diferencias de metodologías, instrumentos y estándares de acreditación (pregrado o posgrado) tienen que ver solamente con la naturaleza de los planes de estudio, ya que se encontrarán variaciones para comprender, por ejemplo, las formas de evaluación de una facultad de artes y una facultad de ingeniería. Todo ello conjuga un detonador en el mejoramiento de la calidad de los servicios educativos que ofrecen las instituciones acreditadas. Es de precisar que existen dos tipos de acreditación: la institucional y la especializada. La primera evalúa la calidad institucional en general y la segunda examina programas específicos de estudio. 


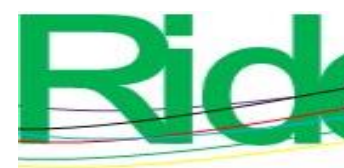

Revista Iberoamericana para la

Investigación y el Desarrollo Educativo

ISSN 2007 - 7467

\section{La acreditación en el contexto nacional mexicano}

En México, se crearon en el año 1991 los Comités Interinstitucionales para la Evaluación de la Educación Superior (Ciees) como organismos de carácter no gubernamental, cuyas principales actividades están encaminadas a la evaluación diagnóstica de programas académicos, funciones institucionales y acreditación de programas y espacios académicos. Posteriormente, en el año 2001, se constituyó el Copaes. Entre sus funciones, destaca la de regular los procesos de acreditación y dar certeza de la capacidad académica, técnica y operativa de los organismos acreditadores. Cabe aclarar que no acredita los programas académicos ni cuenta con posibilidades de castigar a las instituciones que no aprueben el proceso de acreditación, ya que esto corresponde a los organismos acreditadores.

Según Copaes (2019b), "la acreditación es el resultado de un proceso de evaluación y seguimiento sistemático y voluntario del cumplimiento de las funciones universitarias de una IES" que permite obtener información fidedigna y objetiva sobre la calidad de los programas académicos que desarrolla (párr. 1). Permite también certificar ante la sociedad la calidad de los recursos humanos formados y de los diferentes procesos que tienen lugar en una institución educativa. En ese sentido, las IES deben ser las primeras instancias responsables y garantes de la calidad de los programas académicos que cobijan. La acreditación descansa en la búsqueda permanente de la excelencia y representa el esfuerzo colectivo de la comunidad universitaria para rendir cuentas a sí misma y a la sociedad sobre la pertinencia, relevancia y calidad de su ser y quehacer institucional.

La Anuies es otra asociación creada para mejorar la calidad educativa en México; ha participado en la formulación de programas, planes y políticas nacionales, así como en la creación de organismos orientados al desarrollo de la educación superior mexicana. Es una asociación no gubernamental, de carácter plural, que agremia a las principales instituciones de educación superior del país cuyo común denominador es su voluntad para promover su mejoramiento integral en los campos de la docencia, la investigación y la extensión de la cultura y los servicios. Actualmente, está conformada por 203 universidades e instituciones de educación superior tanto públicas como particulares de todo el país (Anuies, 2019).

El Banco Mundial (BM), Unesco, OCDE y el Banco Interamericano de Desarrollo (BID) son cuatro de los organismos internacionales que ejercen una influencia relevante en las políticas educativas de los países subdesarrollados, especialmente en asuntos de la educación superior. Asimismo, han promovido a nivel internacional acciones encaminadas a 


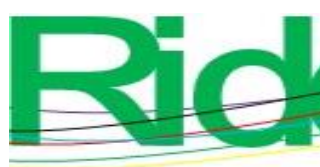

\section{Revista Iberoamericana para la Investigación y el Desarrollo Educativo ISSN 2007 - 7467}

la evaluación externa a fin de formular políticas de mejora y búsqueda de la excelencia. Como ya se ha visto, el sistema de acreditación en México está encabezado oficialmente por el Copaes, aunque también opera otro organismo que cumple en cierto sentido funciones acreditadoras, la Federación de Instituciones Mexicanas Particulares de Educación Superior (Fimpes). En la tabla 1 se muestran los alcances de evaluación para cada uno de ellos.

Tabla 1. Acreditadoras de programas académicos

\begin{tabular}{|l|l|l|}
\hline Carácter de la & Copaes & Fimpes \\
\hline Método de trabajo & Voluntario & Voluntario \\
\hline Procedimiento básico & $\begin{array}{l}\text { Autoevaluación con la } \\
\text { participación de todos los } \\
\text { miembros de la institución. }\end{array}$ & $\begin{array}{l}\text { Autoestudio con y evaluación } \\
\text { participación de los } \\
\text { miembros de la institución } \\
\text { independientes }\end{array}$ \\
\hline $\begin{array}{l}\text { Indicadores de } \\
\text { desempeñon }\end{array}$ & $\begin{array}{l}\text { Evaluación por un organismo } \\
\text { acreditador. }\end{array}$ & $\begin{array}{l}\text { Cada organismo vita. } \\
\text { determina las áreas del } \\
\text { programa académico a evaluar } \\
\text { y cuáles son los respectivos } \\
\text { indicadores. }\end{array}$ \\
\hline
\end{tabular}

Fuente: Elaboración propia con base en Copaes (2019b) y Fimpes (2019)

Ahora bien, la acreditación es el reconocimiento formal y público otorgado por un ente externo a un programa académico que ha logrado avances significativos en el cumplimiento de su misión y objetivos declarados, y satisface así a un conjunto acordado de criterios, indicadores y estándares de pertinencia y calidad establecidos por un organismo acreditador. En la medida en que crecen y se diversifican las actividades y ofertas educacionales, investigativas y de extensión de una institución universitaria, se hace 

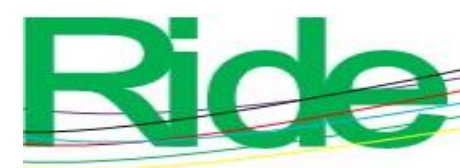

Revista Iberoamericana para la Investigación y el Desarrollo Educativo

ISSN 2007 - 7467

indispensable que existan y operen efectivamente mecanismos de control, cohesión y confiabilidad pública respecto de ellos.

Esta necesidad tiene su máxima expresión en la acreditación, que es realizada por un organismo acreditador, el que a su vez es regulado por una instancia superior. Actualmente, son 30 los organismos que poseen el reconocimiento de Copaes para realizar el proceso de acreditación de programas académicos de IES en diferentes áreas como sociales, medicina, ciencias, tecnología, entre otras.

De manera general, como lo menciona Elías (2015), la acreditación es un mecanismo que encuentra sus raíces en la lógica del mercado. Su principal función es otorgar reconocimiento público a aquellas instituciones y programas educativos que cuentan con un mínimo estándar de calidad.

Para tal efecto, la evaluación se rige por un instrumento denominado Marco General de Referencia, publicado por el Copaes (2016), del cual surgen los lineamientos técnicometodológicos para realizar el proceso de acreditación de un programa académico. En él, se establecen los documentos y pruebas necesarias (denominadas evidencias) para el proceso de acreditación, los atributos a evaluar a través de los indicadores y la definición de cada una de las etapas del proceso. El instrumento coadyuva a la mejora continua de la calidad a partir de los resultados obtenidos.

Actualmente, existen diversas asociaciones dedicadas a la acreditación de programas de estudios de nivel superior; dependiendo de las áreas de conocimiento, es el organismo que otorga la acreditación. En la tabla 2 se presentan algunas de ellas. 
Tabla 2. Organismos acreditadores mexicanos

\begin{tabular}{|c|c|}
\hline Nombre & Sigla \\
\hline Asociación para la Acreditación y Certificación en Ciencias Sociales & Acceciso \\
\hline Asociación Mexicana de Facultades y Escuelas de Medicina & Amfem \\
\hline $\begin{array}{l}\text { Acreditadora Nacional de Programas de Arquitectura y Disciplinas del } \\
\text { Espacio Habitable }\end{array}$ & Anpadeh \\
\hline Comité de Acreditación y Certificación de la Licenciatura en Biología & Caceb \\
\hline Consejo de Acreditación de la Enseñanza en Contaduría y Administración & Caceca \\
\hline Consejo de Acreditación de la Enseñanza de la Ingeniería & Cacei \\
\hline Consejo para la Acreditación de la Educación Superior de las Artes & Caesa \\
\hline Consejo Nacional para la Enseñanza e Investigación en Psicología & CNEIP \\
\hline Consejo para la Acreditación de Programas Educativos en Humanidades & Coapehum \\
\hline Consejo Mexicano para la Acreditación de Enfermería & Comace \\
\hline Consejo Mexicano de Acreditación de la Enseñanza de la Arquitectura & Comaea \\
\hline Consejo Mexicano para la Acreditación de la Educación Farmacéutica & Comaef \\
\hline Consejo Mexicano para la Acreditación de la Educación Médica & Comaem \\
\hline Consejo Mexicano para la Acreditación de Programas de Diseño & Comaprod \\
\hline Comité Mexicano de Acreditación de la Educación Agronómica & Comeaa \\
\hline Consejo Nacional de Acreditación de la Ciencia Económica & Conace \\
\hline $\begin{array}{l}\text { Consejo Nacional de Enseñanza y del Ejercicio Profesional de las } \\
\text { Ciencias Químicas }\end{array}$ & Conaecq \\
\hline Consejo para la Acreditación de la Enseñanza del Derecho & Conaed \\
\hline Consejo Nacional de Educación Odontológica & Conaedo \\
\hline Consejo Nacional para la Calidad de la Educación Turística & Conaet \\
\hline Consejo Nacional de Acreditación en Informática y Computación & Conaic \\
\hline Consejo Nacional para la Calidad de Programas Educativos en Nutriología & Concapren \\
\hline Consejo Nacional de Educación de la Medicina Veterinaria y Zootecnia & Conevet \\
\hline $\begin{array}{l}\text { Consejo Nacional para la Acreditación de la Educación Superior en } \\
\text { Derecho }\end{array}$ & Confede \\
\hline
\end{tabular}

Fuente: Elaboración propia con base en Copaes (2019b) 


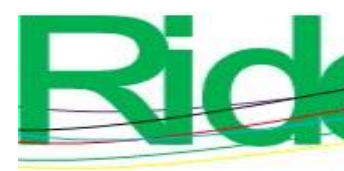

Revista Iberoamericana para la Investigación y el Desarrollo Educativo ISSN 2007 - 7467

La diversidad de los programas académicos de las universidades ha llevado a la creación de estos organismos a fin de contribuir de la mejor manera con la tarea de impulsar las acciones específicas que se deben cumplir para elevar la calidad de la educación superior del país. Así, se procura que los procesos de acreditación en las diversas áreas del conocimiento cuenten con un organismo especializado con la debida pertinencia y heterogeneidad.

En mi opinión, los sistemas de acreditación por sí solos no resuelven los problemas de calidad en la educación. Sin embargo, considero que sí facilita la formulación de estrategias a partir de los resultados de cada uno de los indicadores. En una forma de darle empuje a acciones orientadas a la mejora de la calidad en la educación. No se trata de decir si está bien o está mal, se trata de saber en dónde se está. A más de tres décadas de creados los procesos, comprender cómo la acreditación influye en la calidad de la educación aún no ha permeado lo suficiente y es así que nos encontramos con personas que consideran que una IES acreditada no es sinónimo de calidad.

\section{La acreditación en el contexto internacional}

La evolución en temas de calidad en la educación ha transformado paradigmas de procesos de acreditación nacional a internacional, de procesos a productos (profesionistas), de pregrado y posgrado a transversales. Los organismos acreditadores ahora también deben atender una mayor diversidad de niveles, ámbitos disciplinarios y modalidades de educación, al tiempo que se insertan otros evaluadores como colegios de profesionistas. Esto conlleva a una dificultad que en términos normativos aún está en estudio, ya que no solo aplican los estándares nacionales, sino que requieren de acuerdos y entendimientos internacionales. Rama (2009) menciona que la educación más allá de las fronteras en términos de las nuevas características de internacionalización demanda de una acreditación internacional. Y esto, sin duda alguna, pudiera aunarse a la globalización de muchos otros procesos de carácter cultural, artístico, etc.

La acreditación internacional introduce una cuestión de calidad de servicios educativos desde otros países que deriva en buscar respuestas para identificar lo que se evalúa y acredita, cómo se lleva a cabo y quién lo lleva a cabo, y de manera particular, cuáles serían los impactos. Al respecto, Rama (2009) subraya que, derivado de las demandas de calidad y competitividad, los sistemas de evaluación han evolucionado visiblemente, tal y como se 


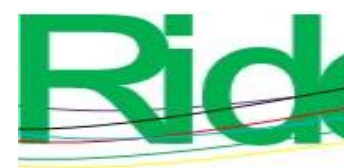

Revista Iberoamericana para la Investigación y el Desarrollo Educativo ISSN $2007-7467$

muestra en la tabla 3. Esta perspectiva pone de manifiesto las transformaciones graduales que son respuesta a las dinámicas del cambio y mejora continua.

Tabla 3. Evolución de los sistemas de evaluación y acreditación

\begin{tabular}{|l|l|}
\hline Desde su creación & Hacia su nueva perspectiva \\
\hline Autoevaluación cualitativa & Autoevaluación cuantitativa \\
\hline Evaluación de insumos & $\begin{array}{l}\text { Evaluación de resultados y exámenes para } \\
\text { el ejercicio profesional }\end{array}$ \\
\hline Preeminencia de la autoevaluación & Mayor valorización del evaluación externa \\
\hline Evaluación centralizada & Procesos de evaluación regionalizados \\
\hline Evaluación académica & Evaluación de múltiples dimensiones \\
\hline Evaluación para el mejoramiento de la & $\begin{array}{l}\text { Evaluación para obtención de recursos } \\
\text { presupuestales }\end{array}$ \\
\hline Evalidad & $\begin{array}{l}\text { Evaluaciones de competencias } \\
\text { profesionales }\end{array}$ \\
\hline Evaluaciones presenciales & $\begin{array}{l}\text { Evaluaciones a través de sistemas } \\
\text { informáticos }\end{array}$ \\
\hline
\end{tabular}

Fuente: Rama (2009)

Otro aspecto de interés en relación con la importancia de la acreditación internacional son los resultados que el Global University Network for Innovation [GUNI] (2017) publica sobre la acreditación de la educación superior en el mundo como garantía de calidad. Es una investigación basada en la opinión de expertos para ayudar a identificar tendencias futuras de aspectos que hoy no se han concretado pero que se están desarrollando. Aporta información para la toma de decisiones, señala posibles líneas de acción sobre las que trabajar en el futuro respecto a temas de acreditación y calidad de la educación en el mundo y su reconocimiento explícito en la sociedad. El reporte refleja los resultados y análisis de 11 preguntas, de las que se extrae para los efectos del presente documento la número cuatro: "Sobre qué tan necesaria es la acreditación internacional". Esta fue la que recibió la más alta puntuación de todas las del cuestionario, lo que confirma la cada vez mayor necesidad de las IES de contar con la acreditación internacional. 


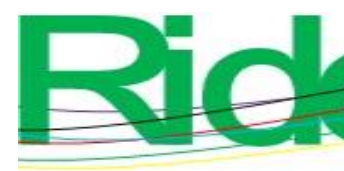

Revista Iberoamericana para la Investigación y el Desarrollo Educativo ISSN 2007 - 7467

En el 2019, GUNI elaboró otro informe que fue presentado en diversos foros para proporcionar a la comunidad académica, los responsables políticos y los encargados de la toma de decisiones dentro de la educación superior un diagnóstico y análisis del estado actual de las cosas, y ofrecer propuestas que pueden ampliar horizontes hacia un enfoque integrado muy necesario para el conocimiento.

\section{La calidad de la educación en el nivel superior}

La posmodernidad se caracteriza por la crítica sustantiva a las formas de actuación de la sociedad en la modernidad, y ello ha conducido a que los grupos sociales busquen caminos alternativos para alcanzar mayores niveles de eficiencia y eficacia en cualquiera de los campos de la actividad humana (De Hoyos, Jiménez y Álvarez, 2015). La educación no ha quedado excluida de tales impactos, y fenómenos como la globalización han tenido diversos efectos en ella. Es así que muchas IES mexicanas han tenido que integrar a sus modelos curriculares diversos elementos que sirvan de patrones de comparación con otros programas de estudio no solo de México sino del mundo. Aunque ello no puede ser unilateral; más bien debe ser derivado del reconocimiento explícito de la sociedad, por lo que es indispensable contar con una acreditación.

Ciertamente, en la actualidad el tema de la educación se ha convertido en un punto central en casi todos los gobiernos, y por ello es un factor de análisis para el desarrollo de los países. El The Global Competitiveness Report 2017-2018 (Schwab, 2018), publicado por el World Economic Forum, es un ejemplo de lo anterior. En él se analizaron 137 economías: México ocupó la posición número 80 en el rubro de educación superior y la 104 en el tema de la calidad del sistema educativo. Contar con un ranking de referencia es importante porque permite establecer comparaciones a partir de diferentes elementos de juicio. Ocupar una posición tan mediocre significa que México debe trabajar arduamente, ya que se cuenta con organismos cuya función es precisamente la de mejorar la calidad educativa.

Por otro lado, en dicho reporte también se evalúan los factores y políticas que determinan el nivel de productividad de una nación, entre las que destacan las siguientes variables:

- Requerimientos básicos (instituciones, infraestructura, ambiente macroeconómico, salud y educación primaria). 


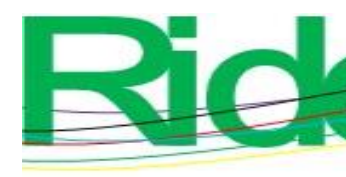

Revista Iberoamericana para la Investigación y el Desarrollo Educativo ISSN 2007 - 7467

- Impulsores de eficiencia (educación superior, capacitación, eficiencia del mercado de bienes y eficiencia del mercado de trabajo, desarrollo del mercado financiero, preparación tecnológica y tamaño del mercado).

- $\quad$ Factores de innovación (sofisticación empresarial e innovación).

El bajo desempeño, de forma constante en algunos países, ha causado preocupación en organizaciones internacionales, tales como la Unesco, que, en conjunto con el BM, el Fondo de Población de las Naciones Unidas (Unfpa), el Programa de las Naciones Unidas para el Desarrollo (PNUD), la Organización de las Naciones Unidas (ONU) y Alto Comisionado de las Naciones Unidas para los Refugiados (Acnur), en el año 2015, organizaron el Foro Mundial sobre la Educación con la participación de más de 160 países.

Uno de los productos más representativos de dicho foro es la Declaración de Incheon y el Marco de Acción para la realización del Objetivo de Desarrollo Sostenible 4 (Unesco, 2016), que expresa diversos compromisos sobre una nueva visión de la educación. Resalta, en el apartado "Hacia 2030”, una nueva visión de la educación del mencionado documento, que la declaratoria 10 pone de manifiesto la importancia de las acreditaciones de los programas educativos, ya que surgen como un reconocimiento público que se hace a la institución educativa en cuestión o bien a un programa o carrera profesional.

Nos comprometemos a promover oportunidades de aprendizaje de calidad a lo largo de la vida para todos, en todos los contextos y en todos los niveles educativos. Ello incluye un mayor acceso en condiciones de igualdad a la enseñanza y formación técnica y profesional de calidad, a la educación superior y a la investigación, prestando la debida atención a la garantía de la calidad. Además, es importante que se ofrezcan vías de aprendizaje flexibles, así como también el reconocimiento, la validación y la acreditación de los conocimientos, habilidades y competencias adquiridos mediante la educación informal y no formal (p. 8).

La calidad en educación debe ser reflejo o producto de diversos componentes que caracterizan a cada institución de educación. Bodero (2014) cita que la calidad educativa se integraría de dos conceptos: primero, la educación como un sistema completo donde existe la diversidad, y que como tal se caracteriza porque contiene múltiples subsistemas fuertemente conectados; es un servicio cuya naturaleza es difícil de describir. En segundo término, se tiene la calidad, que deriva del latín qualitas y que significa 'propiedad o conjunto de propiedades inherentes a una cosa'. 


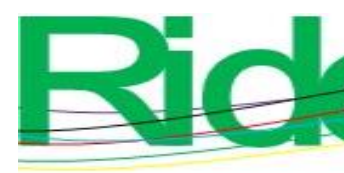

Revista Iberoamericana para la Investigación y el Desarrollo Educativo ISSN 2007 - 7467

Ciertamente, el tema de la calidad educativa tiene que ver más directamente con los resultados esperados de los procesos de educación. En ese sentido, las propuestas y recomendaciones derivadas de la medición de la calidad educativa deben ser incorporadas a las acciones de mejora de los procesos educativos. Es decir, se busca que los nuevos planes de estudio sean producto de las recomendaciones surgidas de los organismos acreditadores. Es así que la calidad educativa está inmersa en el contexto del desarrollo de un país y debiera estar acorde a los modelos de acreditación internacional.

No es que por sí solo el proceso resuelva todos los problemas que se tienen identificados, sino que aporta indicadores numéricos que sirven de punto de partida para la definición de acciones y metas que van orientadas a lograr mejores resultados. Posteriormente, la reacreditación inicia en la revisión de cómo se avanza en las respuestas a las recomendaciones. En muchos de los casos sí se encuentran mejoras, pero desafortunadamente no es parejo el avance en todos los indicadores. Esto dependerá de las posibilidades de cada organismo. Aunque cabe hacer mención que en algunos casos los resultados del proceso solamente sirven para argumentar la gestión de recursos extraordinarios para la IES, sin una mejora real. Esta es una realidad en diversas universidades de nuestro país.

En el documento Revisiones de la OCDE sobre la Evaluación en Educación. México 2012 (Santiago, McGregor, Nushe, Ravela y Toledo, 2012), respaldado por la OCDE, la SEP y el Instituto Nacional para la Evaluación de la Educación (INEE), destaca el papel de la evaluación como instrumento fundamental para lograr la calidad y la equidad en la educación. El informe tiene tres propósitos: “1) proporcionar perspectivas y asesoría a las autoridades educativas mexicanas; 2) ayudar a otros países de la OCDE a entender el enfoque de México y; 3) aportar datos para un informe final comparativo del proyecto" (p. 3).

Como es sabido, la SEP es responsable de la política educativa nacional y de la estrategia general para el sistema educativo. La expansión de dicho sistema educativo en nuestro país, sin duda alguna, ha sido impresionante, aunque, como refiere el mencionado informe, persiste el desafío para fortalecer los componentes del marco de evaluación, de tal forma que aseguren la articulación, coherencia y complementariedad de cara a la mejora.

Ahora bien, como referente en América Latina, desde hace varios años se ha estado trabajando en el tema de la evaluación en la calidad en educación; por citar un ejemplo, se tiene el caso de los integrantes de la Alianza del Pacífico (iniciativa de integración regional), conformada por Chile, Colombia, México y el Perú. En ella, resalta que Perú ha venido 


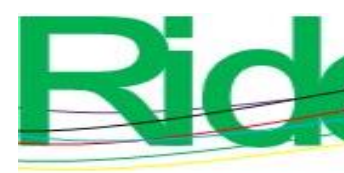

Revista Iberoamericana para la
Investigación y el Desarrollo Educativo
ISSN $2007-7467$

trabajando en la evaluación desde 1980, Chile a partir de 1982, México desde 1988 y Colombia a partir de 1989, por lo que se han acumulado más de 25 años de experiencia en el tema. Como fruto de este trabajo, según el QS Higher Education System Strength Rankings 2018 (QS World University Rankings, 2018), Chile ocupa la posición 32, Colombia la 34, México 31 y Perú figura más allá de la posición 50 de un total de 75 países incluidos.

Esta clasificación evalúa y compara la fortaleza general de la educación superior dentro de cada país y su desempeño en cuatro áreas: fortaleza del sistema educativo, acceso a educación superior de clase mundial, desempeño de la institución educativa líder y el impacto de la inversión nacional que rodea al sistema educativo en cuestión.

En su trabajo, en específico el apartado dedicado a los sistemas de medición y evaluación de la calidad de la educación, Arancibia (1997) formuló sus objetivos de la siguiente forma: generar estándares regionales, establecer un sistema de información y de diseminación de sus avances, desarrollar un programa de investigación sobre las variables de calidad de la educación y fortalecer las capacidad técnica de los ministerios respectivos. En este sentido, contempla que la educación involucra cambios entre los factores y los productos, por lo que debe contar con un sistema de control que permita saber cómo se está operando y si los productos finales se adecuan a los estándares de calidad preestablecidos. Lo anterior implica dos vertientes: por un lado, que se continúe enseñando sin información de lo que se está aprendiendo; por el otro, que se instituya un sistema nacional de medición del rendimiento académico y obtener también información para mejorar el aprendizaje.

Los determinantes de la calidad en educación se refieren principalmente a la dirección y administración, al perfil de los profesores y estudiantes, a los planes de estudio, a las técnicas de enseñanza aprendizaje, a la investigación y su articulación con la docencia, a los laboratorios y talleres y bibliotecas, al manejo de ingresos financiamientos y manejo de recursos. Y también, de forma importante, a la relaciones con otras instituciones, a la vinculación con la sociedad y el intercambio con otras escuelas que se encuentran en el extranjero.

Finalmente, hay que añadir que, tal y como lo resaltan Mendoza y Ortegón (2019), la calidad en la educación es un concepto complejo que tiene que ver con la multidefinición entre los actores de una comunidad universitaria, caracterizada en al menos dos dimensiones: las expectativas como procesos y resultados que pueden ser diferentes según los roles de alumno o docente; y la connotación de la calidad, que es diferente cuando se analiza en fragmentos, ya que estos describen una parte o el resultado del proceso educativo. 


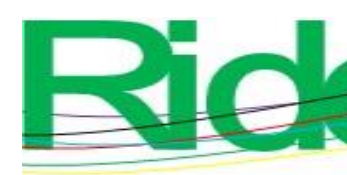

Revista Iberoamericana para la

Investigación y el Desarrollo Educativo

ISSN 2007 - 7467

\section{Propuesta para vincular el trabajo colaborativo y la acreditación internacional a la calidad educativa}

La creación de los organismos acreditadores ha permitido garantizar que los programas académicos cubran estándares de calidad, evidentemente cada uno en su temática específica, pero todos brindando beneficios a las IES y a su comunidad. Algunos de ellos van desde la obtención de recursos económicos, reconocimiento público como instituciones de prestigio, preferencia por los aspirantes para ingresar a un plan de estudios acreditados, certidumbre para los estudiantes de que los contenidos recibidos son actualizados y acordes a las demandas de la sociedad y, por extensión, de que, una vez concluido el programa, serán competitivos en el ámbito laboral, hasta la participación por parte de los empleadores en la reestructuración del plan de estudios que corresponda, y generar con ello interrelación con los empleadores y mejores oportunidades laborales para los egresados.

Las IES en México se desenvuelven en un entorno de competitividad internacional; ocupadas en la certificación de los procesos de enseñanza-aprendizaje, se adhieren a la política educativa del Estado mexicano que busca optimizar la calidad educativa. Derivado de ello, es conveniente cuestionarse acerca del término calidad, así como de los avances y limitaciones de los docentes como factores de la calidad, para conocer las posibilidades que ofrece la calidad educativa a la solución de los problemas de la comunidad.

El rubro que atañe en este documento es el del personal docente como un elemento indispensable en el proceso educativo, del que se evalúan diversos aspectos para determinar si cumple con los requerimientos indispensables de acuerdo a estándares establecidos. Si bien desde principios del siglo XXI la política educativa confirió importancia estratégica a la evaluación como una herramienta imprescindible para la planeación, rendición de cuentas y elaboración de políticas, aún hoy en día se carece de un marco integral que posibilite la conexión de sus componentes. Lo anterior se evidencia todavía más cuando en los últimos años las iniciativas en asuntos de evaluación privilegian la rendición de cuentas más que la mejora en procesos de enseñanza.

Martínez, Tobón y Romero (2017) reportan ciertas problemáticas respecto a la metodología y el modelo general de acreditación, ya que, ante la búsqueda del cumplimiento de la evaluación que conlleva a la acreditación de las IES, en lugar de favorecer las prácticas educativas de calidad, dejan ver que sus procesos son vistos como trámites administrativos que hay que cumplir. 


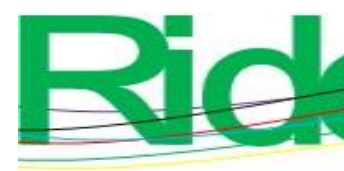

Revista Iberoamericana para la Investigación y el Desarrollo Educativo ISSN $2007-7467$

Asimismo, entre sus sugerencias señalan que es necesario que se promueva el trabajo colaborativo, buscando que se adopte un enfoque formativo, tomando en cuenta la experiencia de los integrantes de la comunidad educativa, además, por supuesto, de la aportación al proceso de acreditación.

Como reflexionan Ferreiro, Brito y Garambullo (2020), el camino por recorrer para lograr que un mayor número de planes de estudio cuenten con la acreditación de un organismo de prestigio internacional aún es largo. Entonces, es fundamental la formulación de estrategias que garanticen que la oferta educativa universitaria sea de calidad, congruente y coherente para participar en los procesos de evaluación y acreditación nacional e internacional, y encaminar acciones hacia esa dirección.

En este mismo orden de ideas, Cervera, Martí y Ríos (2014) dejan en claro que los resultados de una evaluación aportan indicadores para la mejora continua, para ofrecer servicios de más alta calidad. Sin embargo, también discurren en que, ante la complejidad de las metodologías inherentes, aún no se encuentran respuestas para hacer una evaluación formativa y no solo informativa.

Lo anterior abona a la pertinencia de la presente propuesta en el sentido de que los resultados de trabajos colaborativos trasciendan más allá de los autores o participantes directos.

\section{Discusión}

La presente investigación ha sido realizada principalmente con la intención de aportar propuestas para que el trabajo colaborativo entre docentes universitarios innove en los procesos de acreditación tanto nacional como internacional. Ante la pregunta: ¿podría el trabajo colaborativo entre docentes universitarios incidir de manera positiva en los procesos de acreditación de los programas educativos?, la revisión de la literatura ciertamente manifiesta que sí, aunque también se encuentra que hay un largo camino que recorrer. Las fortalezas actuales consisten en que el trabajo colaborativo se viene realizando como respuesta a inquietudes de los docentes que van coincidiendo en el interés de conocer puntos de vista y buenas prácticas en diferentes latitudes de nuestro mundo. Asimismo, se destaca que las nuevas herramientas tecnológicas facilitan su realización.

Evidentemente, para que trasciendan e incluso abonen al tema de la innovación educativa, entendida como el proceso, cambio y mejora educativa que procura optimizar el 


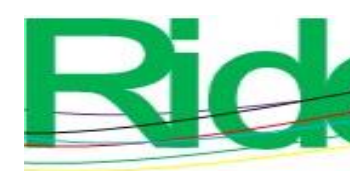

Revista Iberoamericana para la Investigación y el Desarrollo Educativo ISSN 2007 - 7467

aprendizaje de los estudiantes y contribuir a la calidad (Zavala, González y Vázquez, 2019), se debiera poner atención en encontrar formas para su incidencia positiva en los programas educativos de nuestras universidades.

Una de las principales limitaciones del presente trabajo de investigación es que se focaliza en la acreditación de los programas educativos en México. Aquí se comparte la intención de abrir una puerta de interés que atraiga a futuras investigaciones que posibiliten establecer nuevos mecanismos para integrar hallazgos y buenas prácticas en el tema de la acreditación internacional. La generación de estrategias provenientes del trabajo colaborativo entre docentes universitarios y que sean de beneficio de la calidad de los servicios educativos bien pudiera ser uno de los primeros elementos a considerar cuando se planean dichos trabajos.

\section{Premisas para incidir que el trabajo colaborativo entre docentes universitarios trascienda a la acreditación}

Como se ha expuesto, el objetivo principal de la acreditación de las instituciones educativas es fomentar el mejoramiento de la calidad de la educación superior. El proceso conlleva conocer cómo, cuándo, dónde, qué se enseña y qué se aprende; es así que el trabajo colaborativo resulta importante para fortalecer las funciones educativas de docencia, investigación, extensión y vinculación.

En la actualidad, las universidades concentran en la docencia todas las funciones sustantivas, sin embargo, existe la tendencia a separar lo académico de la investigación. Si la investigación tuviera una relación más estrecha con el proceso de enseñanza-aprendizaje, se podría discurrir en un mejoramiento significativo en la calidad de la educación, por lo que se sugiere tomar en cuenta las siguientes consideraciones:

- Muchos de los productos de trabajo colaborativo ni siquiera salen de sus instituciones de origen.

- Sería recomendable publicar los resultados a través las redes de investigación de las universidades nacionales y extranjeras, ya que son un excelente medio de divulgación. No es necesario estar dentro de un cuerpo académico y la visibilidad de los investigadores aumenta.

- Difusión de los productos y logros de la investigación entre los integrantes de la propia comunidad universitaria. 

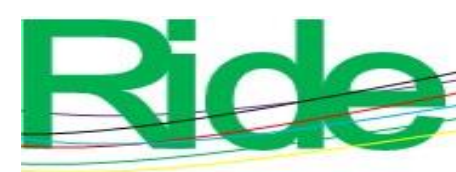

Revista Iberoamericana para la Investigación y el Desarrollo Educativo ISSN $2007-7467$

○ Se debiera fomentar el compromiso de los actores del trabajo colaborativo para permear primeramente entre los propios docentes y alumnos de la institución, por ejemplo, posgrado a pregrado y de investigadores a docentes. Los procesos de acreditación y los de actualización de planes de estudio infaliblemente obtendrían mejores resultados si esta modalidad se convirtiera en una práctica regular. Es decir, con una misma acción se pueden conseguir dos o más efectos en términos de evaluaciones y del impacto en actualizaciones de programas educativos con calidad.

- Lo que se construye en términos de objetivos y resultados de la colaboración académica debiera ser el punto de partida de lo que se traduce en innovación.

- Abordar el tema de innovación requiere articular procesos creativos que lleguen a ser parte del estado cognitivo de un usuario; es contemplada como novedad, independientemente de que se adopte o no (Margalef y Arenas, 2006). Los resultados de trabajos colaborativos acercan a crear o diseñar en congruencia con la realidad social del entorno de las IES.

- La extensión y vinculación universitaria requiere de una integración con los procesos de docencia e investigación.

○ Resulta necesario un trabajo colaborativo con el fin de asegurar la coherencia del contenido de los programas educativos con sus actividades de asesoramiento, consultoría, seguimiento de egresados y vinculación con diversos sectores, impactando también en los resultados de movilidad internacional.

La trascendencia radica en que el objetivo final de una educación de calidad mundial es elevar el nivel de bienestar de los egresados a partir de una mayor competitividad en el mercado laboral, lo cual les permitirá el acceso a mejores ingresos a partir de trabajos especializados, mejor remunerados o de esquemas de emprendimiento viable y sostenible que impacten de manera positiva su vida personal, la de su comunidad y la del país en general. 


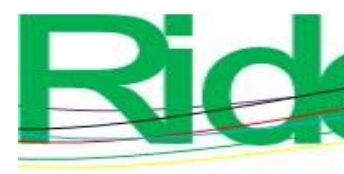

Revista Iberoamericana para la Investigación y el Desarrollo Educativo ISSN $2007-7467$

\section{Conclusiones}

Los factores en los que se concentra el proceso de acreditación para evaluar su calidad son la dirección, la administración, los docentes y estudiantes, el contenido del plan de estudios, los procesos o técnicas de enseñanza y aprendizaje, la difusión, vinculación y extensión, así como la infraestructura y aspectos de financiamiento. Cada uno de ellos se desarrolla en diversos departamentos, y muchas veces sus propios procesos y procedimientos se construyen de manera endógena, sin contar con una visión general y objetivo principal de la institución educativa, centrándose en sus objetivos muy particulares, por lo que algunas dificultades para el cumplimiento de los estándares de calidad tienen que ver precisamente con la necesidad de articular cada uno de los departamentos y áreas con el propósito fundamental de las IES.

Para poder construir procedimientos de calidad bajo un fin común, es imprescindible establecer el trabajo colaborativo como forma de trabajo. Mediante la interacción de las diversas áreas de la organización de la institución educativa, se obtendría una justificada contribución de cada una de ellas al objetivo común, ya que ello significa un verdadero intercambio de información y conocimiento, y no solo un simple reparto de tareas y responsabilidades; un verdadero trabajo colaborativo en el que también se incluye un reconocimiento de las habilidades y una atención adecuada de las actitudes y posturas que se tomen. El trabajo colaborativo en la estructuración y la acreditación de los programas educativos también puede asegurar una mejora significativa en su calidad.

A manera de referencia, en la IES en la que colaboro hace tiempo se inició el fortalecimiento de los programas a partir de resultados de ejercicios colaborativos entre docentes y estudiantes de diversos países. Existen trabajos de titulación y obtención de grado con dichas aportaciones. El interés del presente documento radica en llamar la atención a otras instituciones para integrar los trabajos colaborativos, ya que, en mi experiencia, durante mi participación en diversos procesos de acreditación, el tema de la colaboración entre pares internacionales que abonen a la mejora de los programas educativos ha sido poco explorado. Los estudiantes, a quienes nos debemos, poco o nada conocen de los resultados de dichas colaboraciones. Es así que incluso en sus programas de movilidad conocen escasamente trabajos que pudieran ayudar en su proceso de internacionalización. 


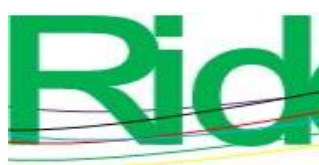

Revista Iberoamericana para la Investigación y el Desarrollo Educativo

ISSN $2007-7467$

Finalmente, es recomendable contar con un esquema de seguimiento de largo plazo y acorde a los parámetros de acreditación internacional para poder identificar, medir y evaluar el impacto de los trabajos colaborativos en la calidad de la educación.

\section{Futuras líneas de investigación}

Sería pertinente para continuar con las aportaciones en esta materia, que se contemplen líneas de investigación que muestren el impacto de los procesos de acreditación en términos de eficiencia terminal y reducción de la deserción estudiantil. Asimismo, sería importante la creación de modelos que faciliten la difusión de los resultados de la colaboración académica internacional para promover la intervención activa de todos los involucrados en la enseñanza aprendizaje y la mejora académica. De tal forma que se refuerce que una IES acreditada es sinónimo de calidad e innovación, responsabilidad de toda la comunidad universitaria. 


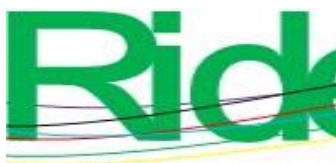

Revista Iberoamericana para la
Investigación y el Desarrollo Educativo
ISSN $2007-7467$

\section{Referencias}

Ángel, I. y Cano, L. (2011). Experiencia de un trabajo colaborativo con estudiantes y docentes de diferentes países mediado por las tecnologías de la información y la comunicación: proyecto colaborativo Interuniversitario, capítulo Colombia. Revista de Educación Comunicación y Tecnología, 6(11), 663-666.

Arancibia, V. (1997). Los sistemas de medición y evaluación de la calidad de la educación. Santiago, Chile: Organización de las Naciones Unidas para la Educación. Recuperado de https://unesdoc.unesco.org/ark:/48223/pf0000183651.

Asociación Nacional de Universidades e Instituciones de Educación Superior [Anuies]. (2017). Acerca de la Anuies. Recuperado de http://www.anuies.mx/anuies/instituciones-de-educacion-superior/.

Bodero, H. (2014). El impacto de la calidad educativa. Apuntes de Ciencia \& Sociedad, 4(1), 112-117.

Bozeman, B. and Craig, B. (2013). Academic faculty in university research centers: neither capitalism's slaves not teaching fugitives. The Journal of Higher Education, 84(1), $88-121$.

Branco, P. and Craig, B. (2010) Influencing scientists' collaboration and productivity patterns through new institutions: University research centers and scientific and technical human capital. Research Policy, 39(5), 610-624. Retrieved from https://doi.org/10.1016/j.respol.2010.02.013.

Cervera, C., Martí, M. y Ríos, D. (2014). Evaluación y acreditación de la educación superior: tendencias, prácticas y pendientes en torno a la calidad educativa. Atenas, 3(27), 115.

Consejo de Acreditación en Ciencias Administrativas, Contables y Afines [Caceca]. (2018).

Acreditación. Recuperado de http://caceca.org.

Consejo para la Acreditación de la Educación Superior [Copaes]. (2016). Marco General de Referencia para los Procesos de Acreditación de Programas Académicos de Tipo Superior (Ver. 3.0). Ciudad de México, México: Consejo para la Acreditación de la Educación Superior. Recuperado de https://www.copaes.org/documentos/Marco_de_Referencia_V_3.0_0.pdf.

Consejo para la Acreditación de la Educación Superior [Copaes]. (2019a). Copaes. Recuperado de https://www.copaes.org/copaes.html. 


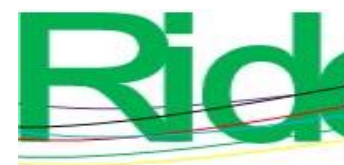

Revista Iberoamericana para la Investigación y el Desarrollo Educativo ISSN $2007-7467$

Consejo para la Acreditación de la Educación Superior [Copaes]. (2019b). Proceso de acreditación. Recuperado de https://www.copaes.org/procesoacreditacion.html.

De Hoyos, J., Jiménez, J. y Álvarez, A. (2015). Los procesos de acreditación en México: efectos en el cambio institucional y la enseñanza de la Arquitectura y las disciplinas del espacio habitable. (reporte final de investigación). Universidad Autónoma del Estado de México, Cerro de Coatepec.

Domínguez, M., Crhová, J. y Molina, R. C. (2015). La investigación colaborativa: las creencias de los docentes de lenguas. Revista Iberoamericana de Educación Superior, 4(17), 119-134.

Dorta, A. J. (2016). Colaboración internacional y visibilidad de la producción científica por las métricas alternativas. Revista Habanera de Ciencias Médicas, 15(6), 859-863. Recuperado de https://www.redalyc.org/pdf/1804/180449695001.pdf.

Elías, G. (2015). Acreditación de la educación superior en México: ¿una solución a las universidades "patito"? $\quad$ Folios, 33-47. http://www.revistafolios.mx/dossier/acreditacionde-la-educacion-superior-enmexico-una-solucion-a-las-universidades-patito

Federación de Instituciones Mexicanas Particulares de Educación Superior [Fimpes]. (2019). ¿Qué es Fimpes? Recuperado de http://www.fimpes.org.mx.

Ferreiro, V., Brito, J. y Garambullo, A. (2020). Modelo de gestión de calidad como estrategia de planeación en procesos de acreditaciones internacionales. RIDE Revista Iberoamericana para la Investigación y el Desarrollo Educativo, 10(20). Recuperado de https://doi.org/10.23913/ride.v10i20.606.

Global University Network for Innovation [GUNI]. (2007). La educación superior en el mundo 2007. Acreditación para la garantía de calidad. ¿Qué está en juego? México: Mundiprensa.

Jiménez, D. (2013). Beneficios de la certificación y acreditación en la educación. Revista Coepes, 3(8).

López, E. A., García, A. B., \& Larios, S. G. (2018). Asociarse o perecer: la colaboración funcional en las ciencias sociales latinoamericanas. REIS Revista española de investigaciones sociológicas, (161), 3-22.

López, E. M. (2015). El conocimiento colaborativo: primer Editatón WikiUNAM. Revista Digital Universitaria, $16(10)$. http://www.revista.unam.mx/vol.16/num10/art83/index.html 

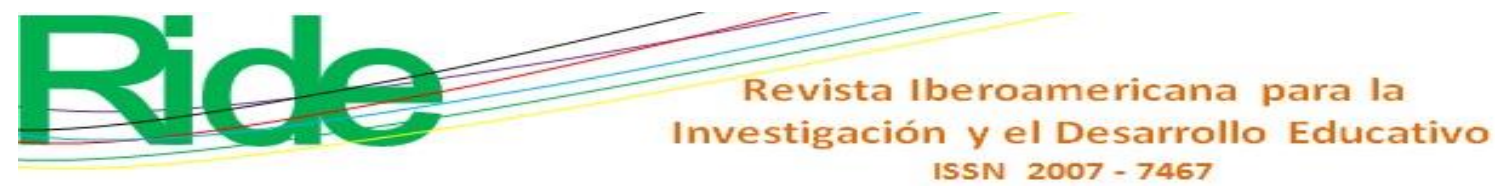

Maldonado, M. (2007). El trabajo colaborativo en el aula universitaria. Laurus, 13(23), 263278. Recuperado de https://www.redalyc.org/articulo.oa?id=76102314.

Maldonado, M. (2008). Aprendizaje basado en proyectos colaborativos. Una experiencia en $\begin{array}{lllll}\text { educación } & \text { superior. } & \text { Laurus, } & 14 & \text { (28), }\end{array}$ https://www.redalyc.org/articulo.oa?id=761/76111716009

Margalef, L. y Arenas, A. (2006). ¿Qué entendemos por innovación educativa? A propósito del desarrollo curricular. Perspectiva Educacional, Formación de Profesores, (47), 13-31. Recuperado de https://www.redalyc.org/articulo.oa?id=3333/333328828002.

Martínez, J., Tobón, S. y Romero, A. (2017). Problemáticas relacionadas con la acreditación de la calidad de la educación superior en América Latina. Innovación Educativa 17(73). Recuperado de http://www.scielo.org.mx/scielo.php?script=sci_arttext\&pid=S166526732017000100079\&lng=es\&tlng=es.

Mendoza, F. y Ortegon, M. (2019). La evaluación en educación superior con fines de acreditación de alta calidad a través de un modelo sistémico con teoría de redes. Revista de la Educación Superior, 48(192), 1-21. Recuperado de https://doi.org/10.36857/resu.2019.192.

Miranda, A. (2019). México nación insignia de la educación superior en Iberoamérica.

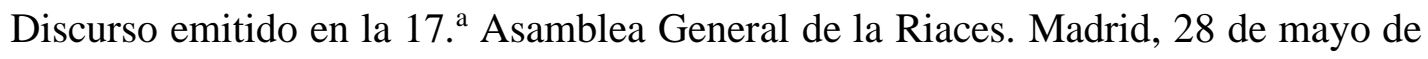
2019. Recuperado de https://www.copaes.org/boletines/comunidad_copaes_no3_junio.pdf.

Organización de las Naciones Unidas para la Educación, la Ciencia y la Cultura [Unesco]. (2015). Declaración de Incheon. Educación 2030. Incheon, Corea del Sur: Organización de las Naciones Unidas para la Educación, la Ciencia y la Cultura. Recuperado de http://unesdoc.unesco.org/images/0023/002338/233813M.pdf.

Organización de las Naciones Unidas para la Educación, la Ciencia y la Cultura [Unesco]. (2016). Educación 2030. Declaración de Incheon y Marco de Acción para la realización del Objetivo de Desarrollo Sostenible 4. Organización de las Naciones Unidas para la Educación, la Ciencia y la Cultura. Recuperado de https://unesdoc.unesco.org/ark:/48223/pf0000245656_spa.

QS World University Rankings. (2018). QS Higher Education System Strength Rankings 2018. Retrieved from https://www.topuniversities.com/system-strengthrankings/2018. 
Rama, C. (2009). El nacimiento de la acreditación internacional. Avaliação (Campinas; Sorocaba), 14(2), 267-290.

Revelo, O. Collazos, C. A. y Jiménez, J. A. (2018). El trabajo colaborativo como estrategia didáctica para la enseñanza/aprendizaje de la programación: una revisión sistemática de literatura. TecnoLógicas, 21(41), 115-134.

Rodríguez, S. M. (2015). Redes de colaboración académica: una respuesta a los desafíos de la globalización y la sociedad del conocimiento. Sinéctica, (44), 1-18.

Santiago, P., McGregor, I., Nusche, D., Ravela, P. and Toledo, D. (2012). Revisiones de la OCDE sobre la Evaluación en Educación. México 2012. Organización para la Cooperación y el Desarrollo Económicos-Secretaría de Educación Pública- Instituto Nacional para la Evaluación de la Educación. Recuperado de http://www.oecd.org/education/school/Revisiones-OCDE-evaluacion-educacionMexico.pdf.

Schwab, K. (ed.) (2018). The Global Competitiveness Report 2017-2018. Geneva, Switzerland: World Economic Forum. Retrieved from http://www3.weforum.org/docs/GCR20172018/05FullReport/TheGlobalCompetitivenessReport2017\%E2\%80\%932018.pdf.

Zavala, M. A., González, I. y Vázquez, M. A. (2019). Modelo de innovación educativa según las experiencias de docentes y estudiantes universitarios. RIDE Revista Iberoamericana para la investigación y el Desarrollo Educativo, 10(20). Recuperado de https://doi.org/10.23913/ride.v10i20.590. 